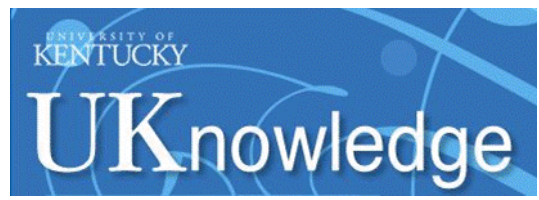

University of Kentucky

UKnowledge

Perinatal Research and Wellness Center Faculty

Publications

Perinatal Research and Wellness Center

$5-2019$

\title{
Associations of First Trimester Co-Use of Tobacco and Cannabis with Prenatal Immune Response and Psychosocial Well-Being
}

\author{
Kristin Ashford \\ University of Kentucky, Kristin.Ashford@uky.edu \\ Amanda Fallin-Bennett \\ University of Kentucky, amanda.fallin@uky.edu \\ Andrea McCubbin \\ University of Kentucky, akmccu0@uky.edu \\ Amanda T. Wiggins \\ University of Kentucky, atwiggins@uky.edu \\ Sheila Barnhart \\ University of Kentucky, sheila.barnhart@uky.edu
}

Follow this and additional works at: https://uknowledge.uky.edu/prwc_facpub

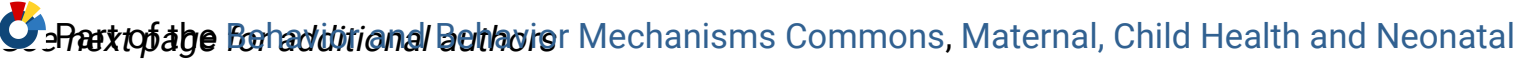
Nursing Commons, Obstetrics and Gynecology Commons, Pediatrics Commons, Psychology Commons, and the Social Work Commons

Right click to open a feedback form in a new tab to let us know how this document benefits you.

\section{Repository Citation}

Ashford, Kristin; Fallin-Bennett, Amanda; McCubbin, Andrea; Wiggins, Amanda T.; Barnhart, Sheila; and Lile, Joshua A., "Associations of First Trimester Co-Use of Tobacco and Cannabis with Prenatal Immune Response and Psychosocial Well-Being" (2019). Perinatal Research and Wellness Center Faculty Publications. 2.

https://uknowledge.uky.edu/prwc_facpub/2

This Article is brought to you for free and open access by the Perinatal Research and Wellness Center at UKnowledge. It has been accepted for inclusion in Perinatal Research and Wellness Center Faculty Publications by an authorized administrator of UKnowledge. For more information, please contact UKnowledge@lsv.uky.edu. 


\section{Associations of First Trimester Co-Use of Tobacco and Cannabis with Prenatal Immune Response and Psychosocial Well-Being}

Digital Object Identifier (DOI)

https://doi.org/10.1016/j.ntt.2019.03.002

Notes/Citation Information

Published in Neurotoxicology and Teratology, v. 73, p. 42-48.

(c) 2019 Elsevier Inc.

This manuscript version is made available under the CC-BY-NC-ND 4.0 license

http://creativecommons.org/licenses/by-nc-nd/4.0/

The document available for download is the authors' post-peer-review final draft of the article.

Authors

Kristin Ashford, Amanda Fallin-Bennett, Andrea McCubbin, Amanda T. Wiggins, Sheila Barnhart, and Joshua A. Lile 
Title

Associations of First Trimester Co-use of Tobacco and Cannabis with Prenatal Immune

Response and Psychosocial Well-Being

Abstract

5 Purpose. This study aims to describe the association of first trimester co-use of tobacco and

6 cannabis with maternal immune response and psychosocial well-being, relative to tobacco use only.

7 Methods. A preliminary midpoint analysis included 138 pregnant women with biologically

8 verified tobacco use, 38 of whom (28\%) also tested positive for recent cannabis use. Maternal

9 perceived stress (Perceived Stress Scale), depressive symptoms (Edinburgh Postnatal

10 Depression Scale), and serum immune markers (IL-1 $\beta$, IL-2, IL-6, IL-8, IL-10, TNF $\alpha$, CRP,

11 MMP8), were collected, although cytokine data were only available for 122 women.

12 Results. Participant average age was 29.1 years, approximately half had a high school education or

13 less, and half were unemployed. Compared to tobacco only users, co-users were more likely to be

14 non-White, younger and more economically disadvantaged. In the adjusted linear regression

15 models, TNF- $\alpha$ levels were significantly lower among co-users relative to tobacco only users, after

16 adjusting for age, race/ethnicity, body mass index and tobacco use group (tobacco cigarettes,

17 electronic nicotine delivery devices [ENDS] or both). TNF- $\alpha$ was the only immune marker found to

18 be significant in this analysis. Measured stress levels $(\mathrm{M}=5.9, \mathrm{SD}=3.3$; potential range $0-16)$ and

19 depression scores $(\mathrm{M}=7.8, \mathrm{SD}=5.8$; potential range $0-30)$ were low across all participants and did

20 not differ as a function of co-use.

21 Conclusion. Preliminary results suggest women co-using during the first trimester exhibit

22 decreased pro-inflammatory immune responsivity on one out of eight markers. Further research is

23 needed to determine the impact of this immune modulation on fetal health outcomes and the

24 unique contribution of cannabis.

25 Key Words: marijuana; nicotine; cytokines; pregnancy; perceived stress 


\section{Introduction}

Tobacco and cannabis are the two most common addictive substances used during pregnancy, and are often used concurrently. Nearly $90 \%$ of cannabis users are also tobacco smokers (Rabin \& George, 2015), and there has been a recent rapid and disproportionate increase in daily cannabis use among female cigarette smokers compared to male smokers (Goodwin et al., 2018). This is of significant concern due to pregnant women being at increased risk of continued use for the duration of their pregnancy (El Marroun et al., 2008; Ko et al., 2015). United States (US) nation-wide survey data reflect that $20 \%$ of pregnant women co-use tobacco and cannabis, (Azofeifa, 2016), with those aged 18-25 years old being more likely to co-use tobacco and cannabis than cannabis alone (Coleman-Cowger, Schauer, \& Peters, 2017). Yet, other recent studies using large databases from individual prenatal clinics indicate that the number of pregnant concurrent users of cannabis and tobacco is considerably higher (approaching 50\%) (Chabarria et al., 2016; Mark, Desai, \& Terplan, 2016).

The consequences of tobacco use during pregnancy have been studied extensively. Nicotine, the primary active constituent of tobacco, is a teratogen and classified as a pregnancy class D drug by the US Food and Drug Administration. Tobacco exposure during pregnancy is associated with numerous adverse physical and psychosocial health effects including, but not limited to, spontaneous preterm birth, small for gestational age infant, placenta previa, placenta abruption, impaired fetal lung and brain development and miscarriage (American College of Obstetricians and Gynecologists, 2017; Castles, Adams, Melvin, Kelsch, \& Boulton, 1999; Centers for Disease Control and Prevention, 2018; Kharrazi et al., 2004; Warren, Albert, Kraft \& Cummins, 2014). Other adverse health effects of prenatal tobacco exposure extend beyond birth and include increased risk for sudden infant death syndrome and numerous respiratory, metabolic, neurobiological and behavioral disorders (e.g. asthma, obesity and attention deficit hyperactivity disorder) (Langely, 
52 Rice \& Thapar, 2005; Maritz \& Harding, 2011; Oken, Levitan \& Gillman, 2008; Weg, Ward, Scarinci,

53 Read, Evans, 2004; Wickstrom, 2007).

54 Prior work is also suggestive of interactions among prenatal tobacco use, immune

55 dysregulation in the mother, and maternal depression and anxiety (Osborne \& Monk, 2013;

56 Coussons-Read, Okun \& Nettles, 2007). High levels of maternal depression and anxiety symptoms

57 are associated with shorter gestation, alterations in fetal neurodevelopment (Schetter \& Tanner,

58 2012) and lower visuospatial working memory performance in the offspring (Buss, Davis, Hobel \&

59 Sandman, 2011).These maternal psychiatric symptoms are also often associated with immune

60 dysregulation in pregnant women, commonly resulting in high circulating serum C-reactive protein

61 (CRP) and proinflammatory cytokines (i.e., interleukin [IL]-6 and tumor necrosis factor [TNF]- $\alpha$ ),

62 and lower levels of the anti-inflammatory cytokine, IL-10 (Christian, Franco, Glaser, \& Iams, 2009;

63 Coussons-Read, Okun, Schmitt, \& Giese, 2005). Further, nicotine directly affects the immune system.

64 In an animal study by Nouri-Shirazi and Guinet (2013), nicotine significantly depressed antibody

65 responses and T-cell proliferation. A study of microglial activation linked nicotine exposure to

66 significantly decreased levels of pro-inflammatory cytokines including interleukin (IL) -6 and TNF-

$67 \alpha$ (Jia et al., 2016). In pregnancy, first trimester tobacco use has been associated with maternal

68 immune dysregulation. For example, significant anti-inflammatory reductions in cervical IL-10

69 were observed in women using tobacco early in pregnancy compared to nonsmokers whereas

70 proinflammatory cytokines (IL-1 $\alpha, 1 \beta, 2,4,6,8$, TNF $\alpha$ ) did not change. (Ashford, O'Brien, McCubbin,

71 Westneat, \& Barnett, 2013; Simhan, Caritis, Hillier, \& Krohn, 2005). An examination of serum

72 cytokines in the second and third trimesters of pregnancy revealed significantly higher

73 concentrations of IL- 6 and IL-1 $\alpha$ among smokers compared to non-smokers (Ashford, Barnett,

74 McCubbin, Kehler, Westneat, 2013). Maternal immune dysregulation is of concern because it has

75 been linked to adverse perinatal outcomes including pre-eclampsia (Ashford et al., 2017) and

76 preterm birth (Goldenberg, Culhane, Iams, \& Romero, 2008; Simhan and Krohn, 2009). 
Within the past two decades, the perceived risk of cannabis use has decreased (Berg et al.,

78 2015; Sinclair, Foushee, Scarinci, \& Carroll, 2013) and public acceptance of cannabis use has

79 increased (Pew Research Center, 2018) in the United States. Perhaps unsurprisingly then, the

80 percentage of national survey respondents reporting past-year cannabis use has also increased

81 (United Nations Office on Drugs and Crime, 2017). Cannabis use in the first trimester of pregnancy

82 has been reported as high as $7.4 \%$, with $16 \%$ of users reporting daily cannabis use (Ko, Farr, Tong,

83 Creanga, \& Callaghan, 2015). Prenatal cannabis use may occur for various reasons including

84 recreation and self-medication (Ko et al., 2015; Park, McPartland, \& Glass, 2004; Wang, Dow-

85 Edwards, Anderson, Minkoff, \& Hurd, 2004). Among women who used cannabis during pregnancy,

86 some endorsed its use as a means to treat nausea and vomiting (Westfall, Janssen, Lucas, \& Capler,

87 2006). Although $\Delta^{9}$ - tetrahydrocannabinol (THC; the primary active constituent of cannabis) is

88 FDA-approved as a treatment for nausea and vomiting associated with cancer chemotherapy, it has

89 not been evaluated for hyperemesis gravidarum, a pregnancy complication resulting in severe

90 nausea, vomiting and alteration in serum electrolytes.

91 The consequences of cannabis use during pregnancy are less clear compared to those of

92 tobacco. Prior research has shown that THC crosses the placenta, although the levels are reduced

93 compared to maternal concentrations (Grant, Petroff, Isoherranen, Stella, \& Burbacher, 2017).

94 Some studies have found adverse outcomes such as increased risk of preterm birth (Burns, Mattick,

95 \& Cooke, 2006), decreased infant head circumference, growth restriction and decreased

96 birthweight (El Marroun et al., 2009; Fergusson, Horwood, \& Northstone, 2002; Metz et al., 2017).

97 However, other studies failed to find negative effects of maternal cannabis use on neonatal

98 outcomes (Conner, Carter, Tuuli, Macones, \& Cahill, 2015; Mark et al., 2016; Shiono et al. 1995; van

99 Gelder et al., 2010). Although some studies suggested initial delays in physical development, all

100 milestones are typically reached on time (Grant et al., 2017). Cognitive impairment has most

101 consistently been linked to fetal cannabis exposure (e.g., Fried \& Watkinson, 2001; Fried, 
102 Watkinson \& Gray, 2003; Huizink \& Mulder, 2006; Willford, Chandler, Goldschmidt \& Day, 2010).

103 For example, prenatal cannabis exposure has been associated with certain deficits in visual and 104 cognitive function in children (Fried \& Watkinson, 2000; Fried, Watkinson \& Gray, 2003) and 105 decreased sustained attention in adolescents (Fried \& Watkinson, 2001). A review of 36 clinical 106 studies found an association between fetal cannabis exposure and conduct disorder, although 107 causality could not be established (Ruisch, Dietrich, Glennon, Buitelaar, \& Hoekstra, 2017).

108 Psychopathological conditions in younger adults, specifically anxiety and depression, are associated 109 with more frequent cannabis use (Hayatbakhsh, Najman, Jamrozik, Mamun, Alati \& Bor, 2007) and 110 co-use use (Ramo, Liu \& Prochaska, 2012).

111 Studies have demonstrated that the endogenous cannabinoid system is a key regulator of

112 immune function, with endogenous cannabinoid agonists, as well as exogenous ligands such as THC,

113 having immunosuppressant effects (reviewed in Olah, Szekanecz \& Biro, 2017). Surprisingly,

114 however, little information is available regarding the impact of prenatal cannabis use or co-use on

115 immune function. Possible epigenetic mechanisms by which maternal cannabis use might impact

116 transgenerational immune function have been proposed (Dong et al., 2019; Zumbrun, Sido,

117 Nagarkatti \& Natarkatti, 2015), but only a single experiment related to maternal cannabis use

118 appears to have been published. In that study, a mouse model was used to demonstrate that

119 prenatal cannabis exposure resulted in T-cell dysfunction in fetal and postnatal animals (Lombard,

120 Hegde, Nagarkatti \& Nagarkatti, 2011).

121 To our knowledge, limited clinical data exist on the consequences of co-use of tobacco and

122 cannabis on maternal or fetal outcomes such as immune function. One recent study reported pre-

123 and postnatal dual exposure to tobacco and cannabis, when compared to tobacco- and cannabis-

124 only groups, increased levels of secretory Immunoglobulin A, an essential antibody for mucosal

125 immunity in early childhood (Molnar et al., 2018). Given that tobacco and cannabis are two of the

126 most widely used substances during pregnancy, and that concurrent cannabis use might confer 
127 additional or synergistic immunity and health risks in pregnant women who use tobacco, this

128 midpoint analysis from an ongoing project sought to describe the effects of first trimester co-use of

129 tobacco and cannabis on serum immune markers (IL-2, IL-6, IL-10, CRP, TNF- $\alpha$ and matrix

130 metalloproteinase [MMP]-8), as well as depression symptoms and perceived stress, compared to

131 tobacco use alone.

132 2.1. Material and Methods

133 This report represents a preliminary midpoint analysis of a larger study to determine the

134 impact of prenatal tobacco use, including electronic nicotine delivery systems (ENDS), on immune

135 response and birth outcomes. Therefore, subject groups consisted of tobacco only users compared

136 to tobacco users who also tested positive for recent cannabis use; a cannabis use only group was

137 not included. An institutional review board (IRB) approved, multisite study using quota sampling

138 was used to meet study aims. Participants were recruited from academic and private prenatal clinics

139 in Kentucky via two methods: 1) women were approached at their obstetric screening

140 appointments; and 2) women proactively responded to posted study flyers. A study nurse

141 determined eligibility based on maternal age (18-44 years); first trimester gestation (less than 14

142 weeks), current tobacco use (within 30 days) and ability to read or write in English. Tobacco use was

143 limited to those who smoked conventional cigarettes and/or any form of ENDS.

144 A research nurse explained the study to eligible participants and obtained informed consent.

145 At enrollment, participants completed a survey (available via hard copy or iPad) that included

146 demographic, tobacco and psychosocial measures. The survey was written at the $6^{\text {th }}$ grade level and

147 took approximately 20 minutes to complete. Survey responses were stored on REDCap, a secure

148 web-based data management system. Following survey completion, study personnel collected urine

149 and serum samples using previously reported methods (Ashford et al., 2017). These biomarkers

150 were used to determine study groupings (tobacco-only and tobacco plus cannabis). Participants

151 were given a $\$ 25$ gift card to a local department store at completion of the study visit. 


\subsubsection{Participants}

Demographic information collected via survey included date of birth, race/ethnicity, partner

154 status, education and income. Age was calculated using the participant's date of birth. Race and

155 ethnicity were assessed separately. First, respondents were asked to indicate whether they were

156 'Hispanic or Latino' or 'Not Hispanic or Latino', and were then asked, 'Which of the following best

157 describes your race?' with response options including 'American Indian/Alaskan Native,' 'Asian,'

158 'Native Hawaiian or Other Pacific Islander,' 'Black or African American,' 'White' and 'More than 1

159 race.' Responses from these two questions were combined and a dichotomous variable ('White, non-

160 Hispanic' or 'Non-white or Hispanic') was used in subsequent analyses. Women were asked to select

161 their partner status from response options including 'Single,' 'Married or living with a partner,'

162 'Divorced or separated,' 'Widowed' or 'Other.' Those who indicated 'Married or living with a partner'

163 were classified as partnered, while all other responses were coded as non-partnered. Employment

164 status was coded as employed ('part-time' or 'full-time') or unemployed ('unemployed,' 'student' or

165 'homemaker'). For education, women were asked 'What is the highest grade or year of school you

166 have completed?' with response options including 'Less than high school graduate,' 'High school

167 graduate or GED,' 'Some college or vocational/trade school' and 'College graduate or beyond.' For

168 analysis, the latter two categories were collapsed to represent beyond high school education. During

169 the first clinic visit (at enrollment), the research nurse recorded height and weight for each

170 participant, which was used to calculate body mass index.

171 Use of conventional and electronic cigarettes was assessed separately. For each product, the

172 research nurse asked 'Have you used e-cigarettes/smoked cigarettes within the last 30 days?'

173 Women who responded 'yes' were coded as current users of the respective product. Those who

174 responded 'yes' to electronic cigarettes were coded as dual or ENDS only users, while those who

175 responded 'no' were coded as conventional cigarette only users. 


\subsubsection{Biological Markers}

Urine and serum samples were collected in the first trimester (8-14 weeks gestation). Urine

179 samples were assayed for the presence of nicotine and cannabis metabolites. Cotinine, a metabolite

180 of nicotine, has a half-life of approximately 9 hours in pregnant women (Bernert et al., 1997;

181 NicAlert, 2007) and was used to confirm tobacco status using a validated commercial assay

182 (NicAlert $($ ). Cotinine levels greater than or equal to $100 \mathrm{ng} / \mathrm{mL}$ validated current tobacco use

183 (Ashford et al., 2010; Bernert, Harmon, Sosnoff, \& McGuffey, 2005). 11-nor-9-carboxy- $\Delta^{9}-$

184 tetrahydrocannabinol (THC-COOH), a major metabolite of THC, was measured using a validated

185 analytical method for measurement of THC-COOH in urine using solid phase extraction and high

186 performance liquid chromatography coupled with negative mode electrospray ionization tandem

187 mass spectrometry. Similar methods have been used previously to assess cannabis use in pregnant

188 women (El Marroun et al., 2010; Westin, Huestis, Aarstad, \& Spigset, 2008). Maternal serum

189 cytokines IL-1 $\beta$, IL-2, IL-6, IL-8, IL-10, TNF $\alpha$, CRP and MMP8 were determined from plasma samples

190 using methods previously reported (Ashford et al., 2017). The iCup Drug Screen (BioScan Screening

191 Systems, Inc., Smyrna, TN) was used to validate illicit drug use (McCarberg, 2011. The iCup employs

192 enzyme-linked immune assays (ELIZA) to detect the presence or absence of the following

193 drugs/drug classes: buprenorphine, morphine/opiates, methadone, oxycodone, benzodiazepines,

194 amphetamines, methamphetamine, cocaine, and THC. An indicator variable for other illicit drug use

195 was created to represent a positive test for any illicit substance use other than cannabis. Only one

196 participant tested positive for alcohol and this participant also tested positive for illicit drug use

197 other than cannabis.

\section{$198 \quad$ 2.1.3. Psychological Measures}

199 Maternal depressive symptoms and perceived stress were measured using tools validated

200 both during and after pregnancy. The 10-item Edinburgh Postnatal Depression Scale was used to

201 measure prenatal depressive symptoms (Gibson, McKenzie-McHarg, Shakespeare, Price, \& Gray, 
2009) and maternal stress was measured using the shortened, 4-item Perceived Stress Scale (PSS)

203 (Glynn, Schetter, Hobel, \& Sandman, 2008; Karam et al., 2012). Both tools have demonstrated

204 consistent reliability throughout pregnancy (EDPS: Cronbach's $\alpha=0.82,0.83$, and 0.84 ,

205 respectively)(Bergink et al., 2011); 4-item PSS with a Cronbach's $\alpha=0.79$ ) (Karam et al., 2012).

$206 \quad$ 2.1.4. Statistical Analysis

Descriptive statistics summarized study variables. The two-sample t-test or chi-square test of association, as appropriate, examined associations among sociodemographic variables and subject group (i.e., co-use of tobacco and cannabis or tobacco use only). Multiple linear regression models were used to determine differences in stress and depression by group, controlling for age,

211 race/ethnicity, partner status, education, income, tobacco use group (conventional cigarette only

212 versus dual or ENDS only user) and other illicit drug use. For the cytokine analysis, the Mann-

213 Whitney U test compared users of both tobacco and cannabis to tobacco only users. Cytokine values

214 were log-transformed as an adjustment for lack of normality in the raw values and multiple linear

215 regression models tested for differences by subject group, adjusting for age, race/ethnicity, body

216 mass index, tobacco use group and other illicit drug use. All analysis was conducted using SAS,

217 version 9.4, with an alpha level of .05 throughout.

\section{3.1. Results}

\section{$219 \quad$ 3.1.1 Sociodemographic Characteristics}

Urine drug tests were performed on 138 tobacco using pregnant women. Overall,

221 participants were primarily white (82\%) and single/not partnered (53\%). The average age was

$222 \quad 29.1$ years; 53\% had a high school education or less and approximately half were unemployed.

223 Approximately one-quarter (24\%) of women self-reported using ENDS, either alone or in

224 combination with cigarettes. Over one-quarter (28\%) of women had a positive urine drug screen

225 for THC-COOH with a median level of $236 \mathrm{ng} / \mathrm{ml}$ (IQR=44-401). Pregnant women who reported co226 use of tobacco and cannabis were younger than tobacco only users $(27.3[\mathrm{SD}=5.0]$ vs 29.8 [SD=5.3] 
227 years old; $t_{(d f=136)}=2.5, p=.02$; Table 1$)$. A higher proportion of co-users defined their race/ethnicity

228 to be other than White $\left(41 \%\right.$ vs $\left.10 \% ; \chi^{2}(d f=1)=16.5, \mathrm{p}<.01\right)$ compared to tobacco only users. In

229 addition, compared to tobacco only users, a greater percentage of co-users listed their job status as 230 unemployed (68.7\% vs $\left.43.3 \% ; \chi^{2}(d f=1)=6.2, p=.01\right)$.

231 Thirty percent of the participants $(n=42)$ were positive for recent use of an illicit substance

232 other than cannabis in the first trimester. The most common substances were methamphetamine $(n$

$233=36)$, prescription opioids $(n=34)$ and cocaine $(n=7)$. There was no difference in the rate of urine

234 drug screens positive for any illicit drug between the groups $(p=.86)$.

235 3.1.2. Cytokine Levels

236 Cytokine data were available for 122 women in the first trimester. In the bivariate analysis,

237 there was a significant difference in TNF- $\alpha(m=2.0 \mathrm{pg} / \mathrm{mL}[\mathrm{IQR}=1.7-2.4] \mathrm{vs} . m=2.4 \mathrm{pg} / \mathrm{mL}$

$\left.238[\mathrm{IQR}=2.0-2.8] ; \chi^{2}(d f=1)=8.0, p=.01\right)$ and $\mathrm{CRP}(m=5.3 \mathrm{mg} / \mathrm{L}[\mathrm{IQR}=1.3-12.9] \mathrm{vs} . m=8.2 \mathrm{mg} / \mathrm{L}$

$239[\mathrm{IQR}=3.0-17.1] ; \chi^{2}(d f=1)=4.6, p=.03$; Table 2$)$ levels between the two groups, with tobacco and

240 cannabis co-users having significantly lower levels compared to tobacco only users for both

241 inflammatory markers, respectively. In the adjusted linear regression models, there was no

242 difference in CRP between groups, while TNF- $\alpha$ levels remained lower among co-users $(b=-0.15$

243 [SE=0.07], $p=.03$ ), adjusting for age, body mass index, race/ethnicity, tobacco use group and other

244 illicit substance use. Because the cytokine values were log-transformed prior to modeling, the

245 geometric mean was interpreted, which indicated that co-users had approximately 14\% lower TNF-

$246 \alpha$ levels compared to tobacco only users $(\exp [$ beta $]=0.86)$. There were no differences by use group

247 for any of the other interleukins or MMP-8 in the unadjusted or adjusted models.

\section{$248 \quad$ 3.1.3. Psychological Measures}

On average, all participants had low stress levels $(\mathrm{M}=5.9, \mathrm{SD}=3.3$; potential range $0-16)$ and

250 depression scores $(M=7.8, S D=5.8$; potential range $0-30)$. There was no significant difference in 
251 perceived stress or depressive symptoms as a function of use group in the unadjusted or adjusted

252 analysis.

\section{4.1. Discussion}

There are well characterized adverse maternal, prenatal and child health effects of tobacco

255 cigarette use during pregnancy. Of concern is the recent escalation in daily cannabis use that has

256 been observed among female cigarette smokers (Goodwin et al., 2018) because concurrent

257 cannabis use might confer additional or synergistic maternal and/or fetal immunity and health

258 risks above those of tobacco. The present midpoint analysis from an ongoing project therefore

259 sought to describe the effects of first trimester co-use of tobacco and cannabis on serum immune

260 markers, as well as depression symptoms and perceived stress, compared to prenatal tobacco use

261 alone. Preliminary results from this analysis suggest that pregnant women co-using tobacco and

262 cannabis during the first trimester have decreased pro-inflammatory immune responsivity as

263 reflected by reduced TNF- $\alpha$ levels. There were no differences in the other seven markers.

264 Little empirical information is available regarding the consequences of co-use of tobacco

265 and cannabis during pregnancy. Analyses of secondary data from a larger study on illicit and

266 prescription drug use during pregnancy indicated that relative to the use of only tobacco or

267 cannabis, co-use was significantly and positively correlated with smaller infant head circumference

268 and birth defects (Coleman-Cowger, Oga, Peters \& Mark, 2018). Similarly, another study found that

269 smaller head size, an increased risk of preterm birth and decreased birth weight in the neonates

270 was associated with prenatal co-use of tobacco and cannabis compared to use of cannabis alone

271 (Chabarria et al., 2016). With respect to childhood effects of co-use of tobacco and cannabis,

272 offspring born to women who reported "decreasing co-use" (i.e., primarily during prenatal and

273 preschool periods) were more likely to be co-users themselves, and children of chronic co-users

274 were more likely to have a substance use disorder, relative to those whose mothers reported no co-

275 use or only postnatal co-use (De Genna, Goldschmidt, Richardson, Cornelius \& Day, 2018). In 
276 addition, a recent study found that pre- and postnatal dual exposure increased secretory

277 Immunoglobulin-A in early childhood relative to tobacco and cannabis-only exposure (Molnar et al.,

278 2018). The present preliminary results extend this limited literature by providing initial evidence

279 that co-use of cannabis and tobacco increases the likelihood of maternal immune system 280 dysregulation relative to the use of tobacco alone.

281 Lower socio-economic status, unemployment, and belonging to a racial minority group are

282 common in women who use cannabis during pregnancy (Chabarria et al., 2016; Conner et al., 2015;

283 Metz et al., 2017; van Gelder et al., 2010). Among tobacco users, co-users of cannabis are more

284 likely to be younger and non-Hispanic Black or Hispanic relative to tobacco only users (Coleman-

285 Cowger et al., 2017), consistent with the current findings. Although demographic characteristics

286 differ between groups, a comprehensive and inclusive approach for identifying and providing

287 cessation interventions should be provided to all co-users of tobacco and cannabis. Future research

288 may also explore the efficacy of interventions tailored to meet the unique needs of distinct

289 demographic groups.

290 Pregnancy is characterized by a physiologic systemic inflammatory response that fluctuates

291 over the course of the pregnancy (Romero, Gotsch, Pineles \& Kusanovic, 2007). Tobacco use during

292 pregnancy is associated with a maternal shift in anti-inflammatory and pro-inflammatory cytokines

293 that can negatively affect fetal outcomes (Ashford et al., 2013; Simhan et al., 2005). To our

294 knowledge, no clinical research has been conducted on the effects of prenatal cannabis use, or co-

295 use of tobacco and cannabis, on maternal, fetal or child cytokine composition. The present study is

296 the first to report that women who co-use tobacco and cannabis exhibit a depressed pro-

297 inflammatory response, as evidenced by significantly lower TNF- $\alpha$ levels, relative to tobacco-only

298 users. TNF- $\alpha$ is a byproduct of macrophages that are responsible for apoptosis, and during

299 pregnancy, are lowest in the first trimester compared to the third trimester (Ashford et al., 2017). 
301 cytokine levels is mixed (Klein, T., Lane, B., Newton, C. \& Friedman, H., 2000), yet largely examines

302 the effects of medical marijuana in patients with chronic inflammatory conditions (e.g. rheumatoid 303 arthritis) (Nagarkatti, P., Pandey, R., Rieder, S., Hegde, V., \& Nagarkatti, M., 2009). In other in-vivo 304 and murine work independently examining cannabis and tobacco, potential effects contributing to 305 TNF- $\alpha$ suppression included the use of unheated THC (Verhoeckx, K. et al., 2006) and higher doses 306 of nicotine (Li-Sha, G. et al., 2015). Further reductions in first trimester TNF- $\alpha$ by the co-use of 307 tobacco and cannabis could compromise the immune system balance between maintaining 308 maternal health and tolerating the semiallogeneic fetus, thereby negatively affecting birth outcomes 309 (Dong et al., 2019). These group differences in TNF- $\alpha$ could be due to the use of cannabis or 310 additive/synergistic effects of tobacco and cannabis in the co-use group, and/or the differing 311 demographic characteristics of the two groups. Further research is needed to uncover the factors 312 driving these group differences.

313 Maternal psychosocial factors such as stress, depression, and anxiety have been linked with 314 tobacco use (Goodwin, Keyes, Simuro, 2007; Hauge, Torgersone, Vollrath, 2012; Zhu \& Valbo, 2002) 315 and cannabis use during pregnancy (Conner et al., 2015; Hayatbakhsh et al., 2007; Mark et al., 2016; 316 Oh, Salas-Wright, Vaughn, \& DiNitto, 2017; Ramo, Liu, Prochaska, 2012), although we are not aware 317 of any studies that have specifically examined the presence of psychiatric disorders in pregnant co318 users. The present midpoint analysis suggests that maternal stress and depressive symptoms do 319 not differ between these groups. Prior studies have compared tobacco-using women and non320 tobacco-using women and found an association between tobacco use and psychological stress and 321 depression (Husky, Mazure, Paliwal, \& McKee, 2008). The present study exclusively recruited 322 tobacco-users, so it is plausible that differences in perceived stress or depressive symptoms were 323 not detected due to the homogeneity of having a sample consisting of all prenatal tobacco users. 
324 Another possibility is that stress and depressive symptom scores were relatively low in both groups

325 making group differences difficult to detect (i.e., a floor effect).

326 Some study limitations warrant mentioning. Weaknesses of this midpoint analysis include

327 the small sample size, the lack of additional comparison groups (i.e., women without exposure to

328 tobacco, and those with cannabis only), and the inability to control for exposures to various

329 medications that might impact study outcomes, such as antibiotics or anxiolytics. Further, there

330 was only one participant with a multiple pregnancy, therefore we were unable to address the

331 potential impact this may have on both psychosocial and immune function. Another limitation is

332 that self-reported cannabis data were not collected. Instead, the presence of urinary THC and level

333 of cotinine (> $100 \mathrm{ng} / \mathrm{mL}$ ) were used for co-use group assignment. Given the varied detection

334 window for urinary THC, it is possible that one or more co-users were misclassified as a tobacco

335 only user, which might have impacted our ability to detect relationships between co-use and

336 maternal outcomes. In addition, the lack of quantitative self-reported use data precluded

337 determination of dose-response relationships. Although standards for the expected concentrations

338 of immune markers at different timepoints during pregnancy have not been established, one

339 possibility is that the varied collection times within the first trimester (weeks 8-13) might have

340 yielded variability in immune markers (Aghaeepour, N. et al., 2017), which also might have

341 impacted our ability to detect relationships between co-use and maternal outcomes. Despite these

342 limitations, the preliminary findings from this midpoint analysis provide the premise for future

343 studies to examine changes in cytokines over the course of pregnancy in women who use tobacco

344 and cannabis.

\section{$345 \quad$ 5.1. Summary and Conclusion}

346 This analysis appears to be the first to compare markers of immune and psychosocial

347 function in first trimester pregnant women who co-use tobacco and cannabis to those in tobacco-

348 only users. These preliminary results suggest that pregnant women co-using tobacco and cannabis 
349 are more likely to be non-White, younger and more economically disadvantaged compared to

350 tobacco-only users. These preliminary results also suggest that co-use in the first trimester is

351 associated with a depressed proinflammatory immune response, as reflected by one immune

352 marker, TNF- $\alpha$. Additional research to measure the range of maternal and fetal immune

353 responsiveness during gestation, as well as long-term follow-up of offspring of women who co-use

354 tobacco and cannabis, is warranted. These findings also support research that includes appropriate

355 comparison groups to disentangle the unique contribution of cannabis use relative to tobacco and

356 cannabis co-use on maternal immune function.

357 Acknowledgement

358 This work was conducted at the University of Kentucky in Lexington, Kentucky, and supported in

359 part by National Institute on Drug Abuse grant number R01DA040694-01 (PI: K Ashford). The

360 content is solely the responsibility of the authors and does not necessarily represent the official

361 views of the National Institutes of Health. Additional support was provided through the University

362 of Kentucky Clinical and Translational Research Center (NIH grant UL1TR001998) REDCap

363 research project database. 


\section{References}

375 Aghaeepour, N., Ganio, E. A., Mcilwain, D., Tsai, A. S., Tingle, M., Van Gassen, S., ... \& Ghaemi, M. S. 376 (2017). An immune clock of human pregnancy. Science immunology, 2(15), eaan2946.

377 American College of Obstetricians and Gynecologists (ACOG). (2017). Smoking cessation during pregnancy. Committee Opinion No. 721. Obstetrics \& Gynecology 130:e200-4.

Ashford, K., Barnett, J., Mccubbin, A., Kehler, S., \& Westneat, S. (2013). Prenatal Systemic Immune Response in Smoking and Nonsmoking Women. Journal of Obstetric, Gynecologic, \& Neonatal Nursing, 42(s1), S82-S83.

Ashford, K., Chavan, N., Ebersole, J., Wiggins, A., Sharma, S., McCubbin, A., Barnett, J., O'Brien, J. (2017). Patterns of Systemic and Cervicovaginal Fluid Inflammatory Cytokines throughout Pregnancy. American journal of perinatology, 35(05), 455-462.

Ashford, K. B., Hahn, E., Hall, L., Rayens, M. K., Noland, M., \& Ferguson, J. E. (2010). The Effects of Prenatal Secondhand Smoke Exposure on Preterm Birth and Neonatal Outcomes. Journal of Obstetric, Gynecologic, \& Neonatal Nursing, 39(5), 525-535. doi: 10.1111/j.15526909.2010.01169.x

Ashford, K., O'Brien, J., McCubbin, A., Westneat, S., \& Barnett, J. (2013). 15: The influence of prenatal smoking status on cervical cytokine distribution. American Journal of Obstetrics \& Gynecology, 208(1), S10.

392 Azofeifa, A. (2016). National estimates of marijuana use and related indicators-National Survey on 393 Drug Use and Health, United States, 2002-2014. MMWR. Surveillance Summaries, 65.

394 Berg, C. J., Stratton, E., Schauer, G. L., Lewis, M., Wang, Y., Windle, M., \& Kegler, M. (2015). Perceived harm, addictiveness, and social acceptability of tobacco products and marijuana among 396 young adults: marijuana, hookah, and electronic cigarettes win. Substance use \& misuse, 397 50(1), 79-89. 
Bergink, V., Kooistra, L., Lambregtse-van den Berg, M. P., Wijnen, H., Bunevicius, R., van Baar, A., \& Pop, V. (2011). Validation of the Edinburgh Depression Scale during pregnancy. Journal Of Psychosomatic Research, 70(4), 385-389.

Bernert, J. T., Harmon, T. L., Sosnoff, C. S., \& McGuffey, J. E. (2005). TECHNICAL NOTE: Use of Cotinine Immunoassay Test Strips for Preclassifying Urine Samples from Smokers and Nonsmokers Prior to Analysis by LCMSMS. Journal of Analytical Toxicology, 29, 814-818.

Bernert, J. T., Jr., Turner, W. E., Pirkle, J. L., Sosnoff, C. S., Akins, J. R., Waldrep, M. K., ... Sampson, E. J. (1997). Development and validation of sensitive method for determination of serum cotinine in smokers and nonsmokers by liquid chromatography/atmospheric pressure ionization tandem mass spectrometry. Clin Chem, 43(12), 2281-2291.

Burns, L., Mattick, R. P., \& Cooke, M. (2006). The use of record linkage to examine illicit drug use in pregnancy. Addiction, 101(6), 873-882.

Buss, C., Davis, E. P., Hobel, C. J., \& Sandman, C. A. (2011). Maternal pregnancy-specific anxiety is associated with child executive function at 6-9 years age. Stress, 14(6), 665-676.

Castles A, Adams EK, Melvin CL et al (1999) Effects of smoking during pregnancy. Five metaanalyses. Am J Prev Med 16:208-215

Centers for Disease Control and Prevention CDC. February 6, 2018. Smoking During Pregnancy. Retrieved on November 21, 2018 from http://www.cdc.gov/tobacco/basic_information/health_effects/pregnancy/index.htm

Chabarria, K. C., Racusin, D. A., Antony, K. M., Kahr, M., Suter, M. A., Mastrobattista, J. M., \& Aagaard, K. M. (2016). Marijuana use and its effects in pregnancy. American Journal of Obstetrics \& Gynecology, 215(4), 506. e501-506. e507.

Christian, L. M., Franco, A., Glaser, R., \& Iams, J. D. (2009). Depressive symptoms are associated with elevated serum proinflammatory cytokines among pregnant women. Brain, behavior, and immunity, 23(6), 750-754. 
423 Coleman-Cowger, V. H., Oga, E. A., Peters, E. N., \& Mark, K. (2018). Prevalence and associated birth

424 outcomes of co-use of Cannabis and tobacco cigarettes during pregnancy. Neurotoxicology

425 and teratology, 68, 84-90.

426 Coleman-Cowger, V. H., Schauer, G. L., \& Peters, E. N. (2017). Marijuana and tobacco co-use among a

427 nationally representative sample of US pregnant and non-pregnant women: 2005-2014

428 National Survey on Drug Use and Health findings. Drug \& Alcohol Dependence, 177, 130-135.

429 Conner, S. N., Carter, E. B., Tuuli, M. G., Macones, G. A., \& Cahill, A. G. (2015). Maternal marijuana use

430 and neonatal morbidity. American Journal of Obstetrics \& Gynecology, 213(3), 422. e421-422.

$431 \quad$ e424.

432 Coussons-Read, M. E., Okun, M. L., \& Nettles, C. D. (2007). Psychosocial stress increases

433 inflammatory markers and alters cytokine production across pregnancy. Brain, behavior,

$434 \quad$ and immunity, 21(3), 343-350.

435 Coussons-Read, M. E., Okun, M. L., Schmitt, M. P., \& Giese, S. (2005). Prenatal stress alters cytokine

436 levels in a manner that may endanger human pregnancy. Psychosomatic Medicine, 67(4),

$437 \quad 625-631$.

438 De Genna, N. M., Goldschmidt, L., Richardson, G. A., Cornelius, M. D., \& Day, N. L. (2018). Trajectories

439 of pre-and postnatal co-use of cannabis and tobacco predict co-use and drug use disorders

$440 \quad$ in adult offspring. Neurotoxicology and teratology, 70, 10-17.

441 Dong, C., Chen, J., Harrington, A., Vinod, K., Hedge, M., \& Hegde, V., (2019). Cannabinoid exposure

442 during pregnancy and its impact on immune function. Cellular and Molecular Life

443 Sciences. February 2019, Volume 76, Issue 4, pp 729-743. https://doi-

$444 \quad$ org.ezproxy.uky.edu/10.1007/s00018-018-2955-0 
El Marroun, H., Tiemeier, H., Jaddoe, V. W., Hofman, A., Mackenbach, J. P., Steegers, E. A., ... Huizink, A. C. (2008). Demographic, emotional and social determinants of cannabis use in early pregnancy: the Generation R study. Drug \& Alcohol Dependence, 98(3), 218-226.

El Marroun, H., Tiemeier, H., Jaddoe, V., Hofman, A., Verhulst, F., van den Brink, W., \& Huizink, A. (2010). Agreement between maternal cannabis use during pregnancy according to selfreport and urinalysis in a population-based cohort: the Generation R Study. European addiction research, $17(1), 37-43$.

El Marroun, H., Tiemeier, H., Steegers, E. A., Jaddoe, V. W., Hofman, A., Verhulst, F. C., ... Huizink, A. C. (2009). Intrauterine cannabis exposure affects fetal growth trajectories: the Generation R Study. Journal of the American Academy of Child \& Adolescent Psychiatry, 48(12), 1173-1181.

Fergusson, D. M., Horwood, L. J., \& Northstone, K. (2002). Maternal use of cannabis and pregnancy outcome. BJOG: An International Journal of Obstetrics \& Gynaecology, 109(1), 21-27.

Fried, P. A., \& Watkinson, B. (2000). Visuoperceptual functioning differs in 9-to 12-year olds prenatally exposed to cigarettes and marihuana. Neurotoxicology and Teratology, 22(1), 1120.

Fried, P. A., \& Watkinson, B. (2001). Differential effects on facets of attention in adolescents prenatally exposed to cigarettes and marihuana. Neurotoxicology and teratology, 23(5), 421430.

Fried, P. A., Watkinson, B., \& Gray, R. (2003). Differential effects on cognitive functioning in 13-to 16-year-olds prenatally exposed to cigarettes and marihuana. Neurotoxicology and teratology, 25(4), 427-436.

Gibson, J., McKenzie-McHarg, K., Shakespeare, J., Price, J., \& Gray, R. (2009). A systematic review of studies validating the Edinburgh Postnatal Depression Scale in antepartum and postpartum women. Acta Psychiatrica Scandinavica, 119(5), 350-364. doi: 10.1111/j.16000447.2009.01363.x 
470

471

472

473

474

475

476

477

478

479

480

481

482

483

484

485

486

487

488

489

490

491

492

493

494

Glynn, L. M., Schetter, C. D., Hobel, C. J., \& Sandman, C. A. (2008). Pattern of perceived stress and anxiety in pregnancy predicts preterm birth. Health Psychology: Official Journal Of The Division Of Health Psychology, American Psychological Association, 27(1), 43-51.

Goldenberg, R. L., Culhane, J. F., Iams, J. D., \& Romero, R. (2008). Epidemiology and causes of preterm birth. The lancet, 371(9606), 75-84.

Goodwin R.D., Keyes K., Simuro N. (2007) Mental disorders and nicotine dependence among pregnant women in the United States. Obstet Gynecol. 2007;109(4):875-83.

Goodwin, R. D., Pacek, L. R., Copeland, J., Moeller, S. J., Dierker, L., Weinberger, A., . . Hasin, D. S. (2018). Trends in Daily Cannabis Use Among Cigarette Smokers: United States, 2002-2014. American journal of public health, 108(1), 137-142.

Grant, K. S., Petroff, R., Isoherranen, N., Stella, N., \& Burbacher, T. M. (2017). Cannabis use during pregnancy: pharmacokinetics and effects on child development. Pharmacology \& therapeutics.

Hauge, L. J., Torgersen, L., \& Vollrath, M. (2012). Associations between maternal stress and smoking: findings from a population-based prospective cohort study. Addiction (Abingdon, England), 107(6), 1168-73.

Hayatbakhsh, M. R., Najman, J. M., Jamrozik, K., Mamun, A. A., Alati, R., \& Bor, W. (2007). Cannabis and anxiety and depression in young adults: a large prospective study. Journal of the American Academy of Child \& Adolescent Psychiatry, 46(3), 408-417.

Huizink, A. C., \& Mulder, E. J. (2006). Maternal smoking, drinking or cannabis use during pregnancy and neurobehavioral and cognitive functioning in human offspring. Neuroscience \& Biobehavioral Reviews, 30(1), 24-41.

Husky, M. M., Mazure, C. M., Paliwal, P., \& McKee, S. A. (2008). Gender differences in the comorbidity of smoking behavior and major depression. Drug and Alcohol Dependence, 93(1), 176-179. doi: https://doi.org/10.1016/j.drugalcdep.2007.07.015 
495

496

497

498

499

500

501

502

503

504

505

506

507

508

509

510

511

512

513

514

515

516

517

518

Jia, J., Peng, J., Li, Z., Wu, Y., Wu, Q., Tu, W., \& Wu, M. (2016). Cannabinoid CB2 receptor mediates nicotine-induced anti-inflammation in $\mathrm{n} 9$ microglial cells exposed to $\beta$ amyloid via protein kinase C. Mediators of inflammation, 2016.

Karam, F., Bérard, A., Sheehy, O., Huneau, M. C., Briggs, G., Chambers, C., . . Koren, G. (2012). Reliability and validity of the 4-item Perceived Stress Scale among pregnant women: Results from the OTIS antidepressants study. Research in nursing \& health, 35(4), 363-375.

Kharrazi, M., DeLorenze, G. N., Kaufman, F. L., Eskenazi, B., Bernert Jr, J. T., Graham, S., ... \& Pirkle, J. (2004). Environmental tobacco smoke and pregnancy outcome. Epidemiology, 660-670.

Klein, T. W., Lane, B., Newton, C. A., \& Friedman, H. (2000). The Cannabinoid System and Cytokine Network (44546). Proceedings of the Society for Experimental Biology and Medicine, 225(1), 1-8.

Ko, J. Y., Farr, S. L., Tong, V. T., Creanga, A. A., \& Callaghan, W. M. (2015). Prevalence and patterns of marijuana use among pregnant and nonpregnant women of reproductive age. American Journal of Obstetrics \& Gynecology, 213(2), 201. e201-201. e210.

Langley, K., Rice, F., \& Thapar, A. (2005). Maternal smoking during pregnancy as an environmental risk factor for attention deficit hyperactivity disorder behaviour. A review. Minerva pediatrica, 57(6), 359-371.

Li-Sha, G., Jing-Lin, Z., Guang-Yi, C., Li, L., De-Pu, Z., \& Yue-Chun, L. (2015). Dose-dependent protective effect of nicotine in a murine model of viral myocarditis induced by coxsackievirus B3. Scientific reports, 5, 15895.

Lombard, C., Hegde, V. L., Nagarkatti, M., \& Nagarkatti, P. S. (2011). Perinatal exposure to $\Delta$ 9tetrahydrocannabinol triggers profound defects in $\mathrm{T}$ cell differentiation and function in fetal and postnatal stages of life, including decreased responsiveness to HIV antigens. The Journal of pharmacology and experimental therapeutics, 339(2), 607-17. 
Maritz, G. S., \& Harding, R. (2011). Life-long programming implications of exposure to tobacco smoking and nicotine before and soon after birth: evidence for altered lung development. International journal of environmental research and public health, 8(3), 875-

523 Mark, K., Desai, A., \& Terplan, M. (2016). Marijuana use and pregnancy: prevalence, associated characteristics, and birth outcomes. Archives of women's mental health, 19(1), 105-111.

McCarberg, B. H. (2011). A critical assessment of opioid treatment adherence using urine drug testing in chronic pain management. Postgraduate medicine, 123(6), 124-131.

Metz, T. D., Allshouse, A. A., Hogue, C. J., Goldenberg, R. L., Dudley, D. J., Varner, M. W., ... Silver, R. M. (2017). Maternal marijuana use, adverse pregnancy outcomes, and neonatal morbidity.

Molnar, D. S., Granger, D. A., Shisler, S., \& Eiden, R. D. (2018). Prenatal and postnatal cigarette and cannabis exposure: Effects on Secretory Immunoglobulin A in early childhood. Neurotoxicology and teratology, 67, 31-36.

533 Nagarkatti, P., Pandey, R., Rieder, S. A., Hegde, V. L., \& Nagarkatti, M. (2009). Cannabinoids as novel

NicAlert. (2007). Expressing the results as cotinine concentration ranges. Retrieved May 2, 2007,

537 Nouri-Shirazi M, Guinet E. Evidence for the immunosuppressive role of nicotine on human dendritic cell functions. Immunology. 2013 [accessed 2017 October 18]; 109(3): 365-373. comparison of trends and correlates among married and unmarried pregnant women. Drug \& Alcohol Dependence, 181, 229-233. 
544 Oken, E., Levitan, E. B., \& Gillman, M. W. (2008). Maternal smoking during pregnancy and child overweight: systematic review and meta-analysis. International journal of obesity, 32(2),

547 Oláh, A., Szekanecz, Z., \& Bíró, T. (2017). Targeting Cannabinoid Signaling in the Immune System: "High"-ly Exciting Questions, Possibilities, and Challenges. Frontiers in immunology, 8, 1487. doi:10.3389/fimmu.2017.01487

Osborne, L. M., \& Monk, C. (2013). Perinatal depression—the fourth inflammatory morbidity of

Park, B., McPartland, J. M., \& Glass, M. (2004). Cannabis, cannabinoids and reproduction. Prostaglandins, leukotrienes and essential fatty acids, 70(2), 189-197.

554 Pew Research Center. (2018). About six-in-ten Americans support marijuana legalization. Retrieved 03/19/18, from http://www.pewresearch.org/fact-

Rabin, R. A., \& George, T. P. (2015). A review of co-morbid tobacco and cannabis use disorders: Possible mechanisms to explain high rates of co-use. The American journal on addictions, 24(2), 105-116.

Ramo, D. E., Liu, H., \& Prochaska, J. J. (2012). Tobacco and marijuana use among adolescents and young adults: a systematic review of their co-use. Clinical psychology review, 32(2), 105-121.

Romero, R., Gotsch, F., Pineles, B., \& Kusanovic, J. P. (2007). Inflammation in pregnancy: its roles in reproductive physiology, obstetrical complications, and fetal injury. Nutrition reviews, 65(suppl_3), S194-S202.

Ruisch, I. H., Dietrich, A., Glennon, J. C., Buitelaar, J. K., \& Hoekstra, P. J. (2017). Maternal substance use during pregnancy and offspring conduct problems: A meta-analysis. Neuroscience \& Biobehavioral Reviews. 
Schetter, C. D., \& Tanner, L. (2012). Anxiety, depression and stress in pregnancy: implications for mothers, children, research, and practice. Current opinion in psychiatry, 25(2), 141.

570 Shiono, P. H., Klebanoff, M. A., Nugent, R. P., Cotch, M. F., Wilkins, D. G., Rollins, D. E., ... Behrman, R. E. The impact of cocaine and marijuana use on low birth weight and preterm birth: A multicenter study. American Journal of Obstetrics \& Gynecology, 172(1), 19-27. doi:

Simhan, H. N., Caritis, S. N., Hillier, S. L., \& Krohn, M. A. (2005). Cervical anti-inflammatory cytokine concentrations among first-trimester pregnant smokers. American Journal Of Obstetrics And Gynecology, 193(6), 1999-2003.

Simhan, H. N., \& Krohn, M. A. (2009). First-trimester cervical inflammatory milieu and subsequent

Sinclair, C. F., Foushee, H. R., Scarinci, I., \& Carroll, W. R. (2013). Perceptions of harm to health from cigarettes, blunts, and marijuana among young adult African American men. Journal of health care for the poor and underserved, 24(3), 1266-1275.

United Nations Office on Drugs and Crime. (2017). World Drug Report 2017. (ISBN: 978-92-1-

van Gelder, M. M., Reefhuis, J., Caton, A. R., Werler, M. M., Druschel, C. M., \& Roeleveld, N. (2010). 148291-1, eISBN: 978-92-1-060623-3). United Nations publication Retrieved from http://www.unodc.org/wdr2017/field/Booklet_5_NEXUS.pdf. 
compound THC-acid have potential immuno-modulating properties not mediated by CB1 and CB2 receptor coupled pathways. International immunopharmacology, 6(4), 656-665.

594 Wang, X., Dow-Edwards, D., Anderson, V., Minkoff, H., \& Hurd, Y. L. (2004). In utero marijuana 595 exposure associated with abnormal amygdala dopamine D2 gene expression in the human fetus. Biological psychiatry, 56(12), 909-915.

597 Warren, G. W., Alberg, A. J., Kraft, A. S., \& Cummings, K. M. (2014). The 2014 Surgeon General's report:"The Health Consequences of Smoking-50 Years of Progress": a paradigm shift in cancer care. Cancer, 120(13), 1914-1916.

Weg, M. W. V., Ward, K. D., Scarinci, I. C., Read, M. C., \& Evans, C. B. (2004). Smoking-related

Westfall, R. E., Janssen, P. A., Lucas, P., \& Capler, R. (2006). Survey of medicinal cannabis use among childbearing women: patterns of its use in pregnancy and retroactive self-assessment of its

Westin, A. A., Huestis, M. A., Aarstad, K., \& Spigset, O. (2008). Short communication: Urinary

Wickström R. Effects of nicotine during pregnancy: human and experimental evidence. Current Neuropharmacology. 2007;5(3):213-222.

612 Willford, J. A., Chandler, L. S., Goldschmidt, L., \& Day, N. L. (2010). Effects of prenatal tobacco, alcohol and marijuana exposure on processing speed, visual-motor coordination, and

615 Zhu SH, Valbo A. Depression and smoking during pregnancy. Addict Behav. 2002;27(4):649-58.

616 Zumbrun, E. E., Sido, J. M., Nagarkatti, P. S., \& Nagarkatti, M. (2015). Epigenetic Regulation of 
Table 1. Sociodemographic characteristics of the study sample

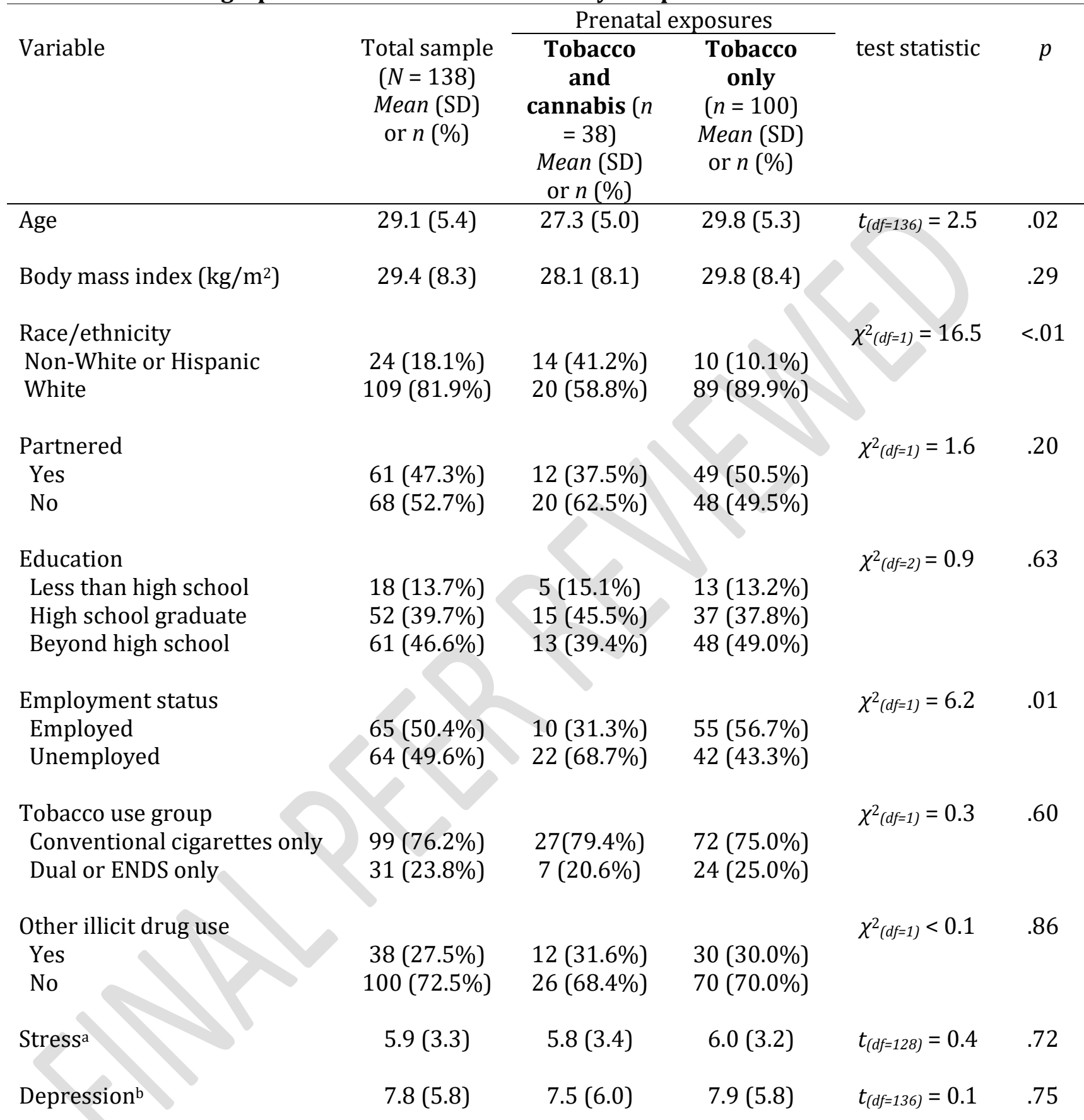

621 Note: Numbers vary due to missing data. Abbreviation: ENDS, electronic nicotine delivery system

622 a Stress measured by the 4-item Perceived Stress Scale; potential scores range from 0-16, with

623 higher scores reflecting more perceived stress

624 b Depressive symptoms measured using the 10-item Edinburgh Postnatal Depression Scale;

625 potential scores range from $0-30$ 
Table 2. Unadjusted and adjusted associations among cytokines and cannabis use

\begin{tabular}{|c|c|c|c|c|}
\hline \multirow[b]{3}{*}{ Cytokine } & \multicolumn{2}{|c|}{ Prenatal exposures } & \multirow[b]{2}{*}{ Unadjusted } & \multirow[b]{2}{*}{ Adjusted } \\
\hline & $\begin{array}{c}\text { Tobacco and } \\
\text { Cannabis } \\
(n=34)\end{array}$ & $\begin{array}{l}\text { Tobacco only } \\
\qquad(n=87)\end{array}$ & & \\
\hline & Median (IQR) & Median (IQR) & $p^{a}$ & $p^{b}$ \\
\hline IL $1 \beta(\mathrm{pg} / \mathrm{mL})$ & $0.09(0.05-0.13)$ & $0.08(0.06-0.11)$ & .36 & .43 \\
\hline IL $2(\mathrm{pg} / \mathrm{mL})$ & $0.13(0.07-0.26)$ & $0.11(0.07-0.22)$ & .59 & .28 \\
\hline IL $6(\mathrm{pg} / \mathrm{mL})$ & $0.74(0.49-1.04)$ & $0.69(0.50-1.12)$ & .95 & .18 \\
\hline IL 8 (pg/mL) & $2.52(2.06-3.38)$ & $2.91(2.28-4.02)$ & .06 & .11 \\
\hline IL $10(\mathrm{pg} / \mathrm{mL})$ & $0.30(0.21-0.42)$ & $0.27(0.22-0.46)$ & .81 & .58 \\
\hline TNF- $\alpha(\mathrm{pg} / \mathrm{mL})$ & $2.03(1.69-2.36)$ & $2.35(1.98-2.75)$ & $<.01$ & .03 \\
\hline $\mathrm{CRP}(\mathrm{mg} / \mathrm{L})$ & $5.34(1.26-12.94)$ & $8.18(3.03-17.05)$ & .03 & .26 \\
\hline MMP 8 (ng/mL) & $27.70(16.01-38.33)$ & $36.33(19.25-60.73)$ & .10 & .76 \\
\hline
\end{tabular}

${ }^{a}$ p-value from Mann-Whitney U test

${ }^{\mathrm{b}} \mathrm{p}$-value from multiple linear regression model adjusting for age, body mass index, race/ethnicity, tobacco use group and other illicit substance use. 